\title{
Empiema torácico en adultos. Aspectos clínicos, microbiología y frecuencia de desenlace quirúrgico
}

\author{
Parapneumonic effusion and thoracic empyema in adults. Clinical aspects, microbiologiy \\ and frequency of surgical outcome
}

\author{
Renata Báez-Saldaña ${ }^{1,2 *}$, Héctor Molina-Corona ${ }^{1,2}$, María E. Martínez-Rendón ${ }^{1,2}$, Marco Iñiguez-García ${ }^{1}$, \\ Araceli Escobar-Rojas ${ }^{2}$ y Teresa Fortoul-Vandergoes ${ }^{2}$ \\ ${ }^{1}$ Instituto Nacional de Enfermedades Respiratorias; '2División de Posgrado, Facultad de Medicina, Universidad Nacional Autónoma de México. \\ Ciudad de México, México
}

\section{Resumen}

Antecedentes: La información sobre el tipo y la frecuencia del tratamiento quirúrgico en los casos de empiema torácico es escasa. Objetivo: Describir las características clínicas, el estudio microbiológico y la frecuencia y el tipo de tratamiento quirúrgico en pacientes adultos con derrame pleural paraneumónico o empiema. Método: Estudio transversal prospectivo de pacientes con diagnóstico de derrame pleural paraneumónico o empiema, de agosto de 2011 a julio de 2014, en un hospital de referencia para enfermedades respiratorias en la Ciudad de México. Se estudiaron las características clínicas, las categōías de riesgo para mal pronóstico en empiema y la frecuencia y el tipo de tratamiento quirúrgico. Resultados: Se estudiaron-284 pacientes cuya mediana de edad fue de 47 años y el 75\% eran hombres. El 57.7\% fueron traslados de otros hospitales. En el $38.8 \%$ de los casos se identificó un microorganismo, con predominio de gramnegativos. Requirieron tratamiento quirúrgico 153 pacientes (53.9\%), de los cuales en el 90\% fue toracotomía con lavado y decorticación. La mortalidad hospitalaria fue del 5.63\%. Conclusiones: La mayor parte de los pacientes llegaron en etapas avanzadas de la enfermedad, y por ello de la mitad requirieron cirugía, de los cuales en el $90 \%$ fue lavado y decorticación. Es deseable favorecer mecanismos para realizar un diagnóstico y un tratamiento tempranos con el fin de disminuir la necesidad de tratamiento quirúrgico.

Palabras clave: Empiema. Microbiología. Tratamiento quirúrgico.

\begin{abstract}
Background: To date, information about the outcome of patients with parapneumonic effusion and empyema is limited. Objective: To describe the clinical characteristics, the microbiological study and the frequency and type of surgical treatment in adult patients with parapneumonic effusion or empyema. Method: A prospective cross-sectional study of patients admitted with parapneumonic effusion or empyema, from August 2011 to July 2014, in a reference hospital for respiratory diseasès in Mexico City, was conducted. Clinical characteristics, microbiology, risk categories for poor prognosis in empyema and frequency and type of surgical treatment were studied. Results: We studied 284 patients whose median age was 47 years, $75 \%$ were men, and $57.7 \%$ were transferred from other hospitals. In $38.5 \%$ of the cases a microorganism was identified and there was a predominance of Gram negative. 153 (53.9\%) required surgical treatment, of which 90\% were thoracotomy with decor-

Correspondencia:

*Renata Báez-Saldaña

Tlalpan, 4502

Col. Sección XVI

Fecha de recepción: 26-08-2019

C.P. 14080 , Ciudad de México, México

E-mail: baezrd@unam.mx

0009-7411/0 2020 Academia Mexicana de Cirugía Publicado por Permanyer. Este es un ar (http://creativecommons.org/licenses/by-nc-nd/4.0/).
\end{abstract}


tication. Hospital mortality was 5.63\%. Conclusions: Most of the patients arrived in advanced stages of the disease, so more than half required surgery, of which $90 \%$ was decortication. It is desirable to favor mechanisms for early diagnosis and treatment to reduce the need for surgical treatment.

Key words: Empyema. Microbiology. Surgery treatment.

\section{Introducción}

Los pacientes con neumonía adquirida en la comunidad pueden desarrollar derrame pleural, cuya frecuencia en la literatura es muy variable. Un estudio de Chalmers, et al. ${ }^{1}$ la describe en el $7.2 \%{ }^{1}$, mientras que otros trabajos, como el de Falguera, et al. ${ }^{2}$, la cifran en un $19 \%$, y el de Feller-Kopman y Light $^{3}$ en el $40 \%$. De los casos que desarrollan derrame pleural por neumonía, una minoría se complican con empiema pleural, el cual se asocia con unas considerables morbilidad y mortalidad, que pueden disminuir de manera ostensible si el cuadro se reconoce y se trata tempranamente. En los últimos años ha habido un aumento en la incidencia de las infecciones de la pleura en adultos, como así lo demuestran estudios realizados en Finlandia ${ }^{4}$, Dinamarca ${ }^{5}$, Canadá ${ }^{2}$ y los Estados Unidos de América?.

En adultos, un estudio en población canadiense observó un aumento estadísticamente significativo de 1.2 veces en la incidencia de empiema durante un periodo de 9 años, entre 1995 y 2003 ${ }^{4}$, y otro estudio en los Estados Unidos de América mostró un incremento de más de seis veces en la tasa de mortalidad por empiema durante un periodo de 4 años, entre 2000 y 2004, en comparación con la tasa de 1950-1975 .

El tratamiento actual del empiema incluye antibióticos y drenaje del espacio pleural mediante la colocación de una sonda endopleural con o sin el empleo de trombolíticos ${ }^{8}$. Este tratamiento falla en el $30 \%$ de los casos, en los que es necesario realizar un abordaje quirúrgico, ya sea toracoscopia videoasistida, lavado de la cavidad y decorticación, o drenaje abierto de la cavidad'.

En un estudio que se realizó en pacientes mexicanos con empiema se identificó que la demora en la toma de decisiones y el tiempo entre el inicio del padecimiento y la decisión del tratamiento fueron los principales factores que condicionaron un evento adverso, que fue desde la prolongación de la estancia hospitalaria hasta la muerte ${ }^{10}$. En México, el empiema sigue siendo una de las patologías pleurales frecuentes y que ocasionan morbilidad y mortalidad significativas; sin embargo, se dispone de información limitada sobre el desenlace de los pacientes con esta enfermedad. El objetivo del presente trabajo fue describir las caraeterísticas clínicas, el estudio microbiológico y la frecüencia y el tipo de tratamiento quirúrgico en pacieñtes adultos con empiema torácico.

\section{Método}

El estudio fue aprobado por el Comité Institucional de Ética en Investigación con el número C11-11 Se realizó un estudio transversal prospectivo de 284 pacientes consecutivos a partir de 15 años de edadicon diagnóstico de derrame pleural paraneumónico y êmpiema (DPP/E), de 2011 a 2014, en un hospitabde referencia para enfermedades respiratorias en la Ciudad de México. Se excluyeron los casos con derrāme pleural por neumonía nosocomial.

A todos los pacientes se les practicaron tomogrăfía helicoidal de tórax y toracocentesis dentro de lasprimeras 24 horas después del ingreso. A los pacientes que llegaron por envío de otro hospital y que ya traían colocada una sonda endopleural no se les realizó toracocentesis. La muestra de líquido pleural seóobtuvo bajo condiciones asépticas y se procesó de forrma estandarizada por el laboratorio de la institución. Se evaluó el aspecto macroscópico del líquido pleurral y posteriormente se envió la muestra en jeringaseestériles de $20 \mathrm{ml}$ para cultivo de microorganismos aerobios, micobacterias y hongos. Así mismo, de manera sistemática, la muestra se procesó para estudio citológico y análisis bioquímico, que incluyó difereñcial de la celularidad, medición del pH, deshidrogenăsa láctica, adenosina desaminasa, glucosa y proteínas. No se realizó medición de la proteína $C$ reactiva in la procalcitonina en el líquido pleural, debido a gue en nuestra institución no son mediciones habituăles para el estudio y el tratamiento de los pacientes con DPP/E. En los casos de empiema con líquido pleural viscoso, la muestra se envió solo para estưdio microbiológico.

El DPP se definió como aquel con características de exudado y con celularidad con predomino de trófilos polimorfonucleares, debido a neumonía. Los 
criterios aceptados para el diagnóstico de empiema torácico, independientemente de su origen, fueron:

- Pus franca al momento de la toracocentesis o hallazgo de microorganismos con la tinción de Gram o cultivo, o todas las siguientes pruebas positivas en el líquido pleural: $\mathrm{pH}<7.2$, glucosa $<60 \mathrm{mg} / \mathrm{dl}$, deshidrogenasa láctica $>1000 \mathrm{Ul} / \mathrm{ml}$, $>3 \mathrm{~g} / \mathrm{ml}$ de proteína, y leucocitos totales de 15,000 células $/ \mathrm{mm}^{3}$.

- Signos físicos, radiológicos y de laboratorio compatibles con un cuadro clínico sugestivo de empiema ${ }^{11}$.

Se consideraron como variables desenlace la cirugía (tipo de cirugía) y la defunción. Las variables independientes fueron las características generales, clínicas (comorbilidad, síntomas, tiempo del padecimiento) y radiológicas; el tipo de empiema, primario o posneumónico o empiema secundario (posneumonectomía, hemotórax, traumatismo, rotura esofágica o secundario a derrame pleural maligno); la presencia y el número de loculaciones; la obesidad (índice de masa corporal $[\mathrm{IMC}]>30 \mathrm{~kg} / \mathrm{m}^{2}$ ) o la desnutrición (IMC < $18.5 \mathrm{~kg} / \mathrm{m}^{2}$ para mujeres y para hombres $\left.<20 \mathrm{~kg} / \mathrm{m}^{2}\right)^{12}$; las pruebas de laboratorio y microbiología; el tiempo entre el inicio del padecimiento y la decisión del tratamiento; la referencia de otro hospital y los microorganismos aislados en el líquido pleural.

La evaluación microbiológica incluyó muestras de líquido pleural para tinción de Gram y cultivo para microorganismos aerobios, expectoración, hemocultivo y de lavado bronquial por fibrobroncoscopia. La indicación para realizar cada uno de los estudios anteriores se individualizó de acuerdo con el caso, excepto para el cultivo de las muestras del líquido pleural, para el que se trató de obtener material en todos los casos. La detección de patógenos respiratorios se realizó de acuerdo con los métodos estandarizados y las guías microbiológicas en el laboratorio de la institución, que cuenta con la certificación de sus métodos. En ningún caso se utilizó microbiología molecular para confirmar la etiología.

Todos los pacientes se trataron con antibióticos intravenosos con un doble esquema que incluyó, en la mayor parte de las ocasiones, un betalactámico y otro fármaco para cubrir anaerobios, como clindamicina o piperacilina-tazobactam, administrados por vía intravenosa durante toda la estancia hospitalaria o hasta completar 3-4 semanas de tratamiento. En todos los casos en los que se obtuvo pus al momento de la toracocentesis, o en caso de DPP con $\mathrm{pH} \leq 7.2$, se colocó sonda endopleural para el drenaje de la cavidad. En ningún paciente se utilizaron fibrinolíticos intrapleurales debido a que es un tratamiento no disponible en la institución. Si no se documentó un drenaje satisfactorio o el paciente no mejoró, ni pớ la clínica ni radiográficamente, el médico neumólogoưtratante valoró, en conjunto con el cirujano de tórax, la decisión de tratamiento quirúrgico, cuya técnicæ de abordaje la tomó el cirujano.

El seguimiento de los casos estudiados fue hầsta el egreso hospitalario, ya fuera por mejoría o־̣por defunción.

Se realizó estadística descriptiva. Las variables continuas se expresaron como mediana (intervalö intercuartílico 25-75 [IIC]) y las variables discretas dicotómicas o politómicas se resumieron cợo frecuencia y porcentaje. Se utilizó el paquete estad́ístico STATA 15.1 (College Station, Texas, USA).

\section{Resultados}

Se estudiaron 284 pacientes cuya mediana de edad fue de 47 años, el $75 \%$ eran hombres y predominó el nivel socioeconómico bajo (89.4\%). El $60 \%$ de là̃población estudiada refirió exposición actual o pasada a tabaquismo, con una mediana de índice tabáquico de 5.3 paquetes-año. El $39 \%$ de los casos refiriónexposición al humo de leña, con una mediana de ínđice de exposición de 45 horas al año. El $82 \%$ de la papoblación presentó alguna comorbilidad; las enfermedades más frecuentes fueron diabetes $(35.5 \%$ e hipertensión arterial sistémica (22.9\%). La mayor parte de los casos había recibido antibiótico previó al ingreso $(72.9 \%)$ y más de la mitad $(57.7 \%)$ fueron traslados de otros hospitales (Tabla 1).

Los síntomas fueron muy variables, pero los que se refirieron con más frecuencia ( $>60 \%$ ) fueron fiebre al interrogatorio, diaforesis, tos con expectoración, đdisnea, dolor torácico y pérdida de peso. En el $35.6 \%$ de los pacientes se registró una presión arterial diastólica $<60 \mathrm{mmHg}$. El $60.5 \%$ presentaron una frecúencia cardiaca $>90$ latidos por minuto y el $15 \%$ tuna frecuencia respiratoria $>30$ respiraciones por minuto. El $52 \%$ presentaron una saturación de oxígeno $<90 \%$. La mediana del tiempo del padecimiento actual fue de 24 días y la mediana de la estancia hospitalaria fue de 19 días. Hubo 16 (5.63\%) defunciones por empiema durante la hospitalización, de las cuales šolo una fue por tuberculosis (Tabla 2).

Casi la mitad de los pacientes presentaron leucocitos $>12,000 \mathrm{~mm}^{3}$ y el $66.5 \%$ tenía neutrofilia. La cocitopenia y la neutropenia fueron poco frecuentes, 
Tabla 1. Características generales y antecedentes al ingreso en los 284 pacientes con derrame pleural paraneumónico o empiema

\begin{tabular}{lc}
\hline Características y antecedentes & $\mathbf{n}(\%)$ \\
\hline Edad en años, mediana (IIC) & $47(36-58)$ \\
Edad en años, intervalo mínimo-máximo & $15-96$ \\
Edad en años estratificada & \\
$15-29$ & $36(12.7)$ \\
$30-39$ & $57(20)$ \\
$40-59$ & $123(43.3)$ \\
$60-69$ & $40(14.1)$ \\
$70-96$ & $28(9.9)$ \\
Sexo masculino & $213(75.00)$ \\
Nivel socioeconómico bajo & $254(89.44)$ \\
Tabaquismo actual o pasado & $172(60.56)$ \\
Índice tabáquico, mediana (IIC) & $5.3(2.0,16.0)$ \\
Alcoholismo (n=279) & $111(39.78)$ \\
Exposición al humo de leña & $108(39.27)$ \\
Índice de exposición al humo de leña & $45(14.5-90)$ \\
(horas/año), mediana (IIC) & \\
Consumo de drogas (n = 284) & $23(8.10)$ \\
Alguna comorbilidad & $234(82.39)$ \\
Diabetes & $101(35.56)$ \\
Hipertensión arterial sistémica & $65(22.89)$ \\
Obesidad (IMC $\geq 30)$ & $50(17.60)$ \\
Cáncer de pulmón & $12(4.23)$ \\
Ingreso previo por empiema & $44(15.49)$ \\
Antibiótico previo al ingreso & $207(72.88)$ \\
Traslado de otro hospital & $164(57.75)$ \\
\hline IC. intervalo intercuartílico 25-75: IMC: índice de masa corporal & \\
&
\end{tabular}

IIC: intervalo intercuartilico 25-75; IMC: índice de masa corporal

y la linfocitopenia (linfocitos $<1000 / \mathrm{mm}^{3}$ ) se documentó en el $26 \%$ de los casos. La anemia y la hipoalbuminemia (hemoglobina < $3 \mathrm{~g} / \mathrm{dl}$ ) fueron frecuentes; las presentaron un $49.6 \%$ y un $80 \%$ de los pacientes, respectivamente (Tabla 3 ).

Los resultados del estudio bioquímico del líquido pleural concordaron con el diagnóstico de DPP/E, con $\mathrm{pH}$ ácido, valores de glucosa disminuidos y cifras de deshidrogenasa láctica muy elevadas. En lo que respecta a las características del DPP/E, la formación de lóculos fue muy frecuente $(80.6 \%)$, con un número de ellos desde 1 hasta 5 , y más de la mitad de los pacientes presentaron dos o más. En el $88 \%$ se documentó paquipleuritis. Se obtuvo pus al momento de realizar la toracocentesis en 140 (56.7\%) de los 247 casos. El $97.5 \%$ de los pacientes tuvieron una categoría de riesgo para mal pronóstico de empiema entre moderado y elevado. La mayor parte de los casos fueron por DPP (37.3\%) y empiema primario (36.6\%) (Tabla 4).

Los microorganismos que se identificaron en el líquido pleural fueron extremadamente variables y solo tenemos datos de los aerobios. En 209 (73.6\%) de 284 casos se realizó cultivo del líquido pleural, y de
Tabla 2. Tipo y frecuencia de los síntomas referidos en el padecimiento actual

\begin{tabular}{|c|c|}
\hline Signos y síntomas & $\begin{array}{l}\text { Población total } \\
\mathrm{N}=284 \mathrm{n} \% \text { ( })\end{array}$ \\
\hline Fiebre al interrogatorio & $197(69.4)$ \\
\hline Calosfríos & $147(51.8)$ \\
\hline Diaforesis & $177(62.3)$ \\
\hline Tos & $234(82: \overline{4})$ \\
\hline Expectoración & $207(72: 9)$ \\
\hline Disnea & $242(85.2)$ \\
\hline Dolor torácico & $235(82.8)$ \\
\hline Hemoptisis o hemoptoicos & $52(18 . \overline{3})$ \\
\hline Ortopnea & $48(16.98$ \\
\hline Pérdida de peso & $172(60.6)$ \\
\hline Mialgias o artralgias & 55 (19.整 \\
\hline Cefalea & $74(26)$ \\
\hline Fatiga & $150(52 . \overline{8})$ \\
\hline Náuseas o vómitos & $80(28.48)$ \\
\hline Confusión & $21(7.45$ \\
\hline Sibilancias & 85 (29.9) \\
\hline Hemitórax afectado & $155(54 . \overline{6})$ \\
\hline Derecho & $120(42.3)$ \\
\hline Izquierdo & $6(2.1) \underline{e}$ \\
\hline Bilateral & $13(4.58)$ \\
\hline Presión arterial sistólica $<90$ mm/Hg & $101(35.56)$ \\
\hline Presión arterial diastólica < 60 mm/Hg & $69(24.3)$ \\
\hline Frecuencia cardiaca > 110 latidos por minuto & $42(14.84)$ \\
\hline $\begin{array}{l}\text { Frecuencia respiratoria }>30 \text { respiraciones } \\
\text { por minuto }\end{array}$ & $121(51.93)$ \\
\hline Saturación de oxígeno < 90\% & $26(13-42)$ \\
\hline $\begin{array}{l}\text { Tiempo del padecimiento (días), mediana (IIC) } \\
(\mathrm{n}=279)\end{array}$ & $19\left(14-\frac{26}{0}\right)$ \\
\hline Días de estancia hospitalaria, mediana (IIC) & $16(5.63)$ \\
\hline Defunción & 它 \\
\hline IIC: intervalo intercuartilico 25-75. & $\begin{array}{l}\text { 흐 } \\
\text { 을 } \\
\text { 은 }\end{array}$ \\
\hline
\end{tabular}

ellos, en $81(38.8 \%)$ casos se aislaron 89 patógenos, de los cuales el $38.5 \%$ fueron grampositivos y el $\stackrel{4}{4} \%$ fueron gramnegativos. Se aisló Mycobacterium tự̆erculosis en el $13.2 \%$ y se identificaron hongos en el $4.3 \%$. Los grampositivos más frecuentes fueron Ştaphylococcus aureus y Streptococcus constellatus, y entre los gramnegativas predominaron las enterobacterias, como Escherichia coli (Tabla 5).

En 153 (53.9\%) casos se llevó a cabo un procedimiento quirúrgico, y de estos, en $138(90.20 \%)$ el procedimiento fue toracotomía para lavado y decörticación. La pleurotomía abierta se practicó en 12 
Tabla 3. Resultados seleccionados de laboratorio clínico en los pacientes con derrame pleural paraneumónico y empiema

\begin{tabular}{|c|c|}
\hline \multicolumn{2}{|l|}{ Parámetros* } \\
\hline Leucocitos $/ \mathrm{mm}^{3}$ & $11,600(8,845-16,750)$ \\
\hline Leucocitos > 12,000/mm³, n (\%) & $129(45.42 \%)$ \\
\hline Leucocitos $<4,000 / \mathrm{mm}^{3}, \mathrm{n}(\%)$ & $4(1.41 \%)$ \\
\hline Neutrófilos $/ \mathrm{mm}^{3}$ & $9,000(6,400-13,620)$ \\
\hline Neutrófilos > 7,500/mm³ , n (\%) & $189(66.55 \%)$ \\
\hline Neutropenia < 2,000 mm³, $\mathrm{n}(\%)$ & $3(1.06 \%)$ \\
\hline Linfocitos totales & $1300(940-1,800)$ \\
\hline Linfocitopenia < 1,000 mm³, $\mathrm{n}(\%)$ & $74(26.06 \%)$ \\
\hline Hemoglobina (g/dl) & $11.8(10.4-13.3)$ \\
\hline Anemia $^{\dagger}, \mathrm{n}(\%)$ & $141(49.6 \%)$ \\
\hline Albúmina (g/dl) & $2.4(2.01-2.83)$ \\
\hline Albúmina $<3 \mathrm{~g} / \mathrm{dl}$ & $216(80.30 \%)$ \\
\hline Deshidrogenasa láctica & $183(145-258)$ \\
\hline
\end{tabular}

(7.84\%) casos y la toracoscopia videoasistida se realizó en $3(1.96 \%)$ casos. La mediana (IIC) del tiempo transcurrido entre el ingreso del paciente y el día en que se practicó la cirugía fue de 8 (4-14) días, y entre la realización de la cirugía y el egreso hospitalario fue de 11 (8-20) días (Tabla 6).

\section{Discusión}

En el presente estudio describimos las características clínicas y la frecuencia y el tipo de tratamiento quirúrgico de pacientes con DPP/E, patología aún prevalente en nuestro medio y que en los países desarrollados ha mostrado un incremento en su incidencia y mortalidad ${ }^{4-7}$. Es el primer estudio prospectivo con un elevado número de pacientes consecutivos con infección pleural de características similares en un centro de investigación en México. La población de pacientes se caracterizó por estar integrada por adultos en etapa productiva, con predominio de los hombres $(75 \%)$, con una frecuencia elevada de exposiciones a tabaquismo o humo de leña, y con al menos una enfermedad concomitante en el $80 \%$ de la población (las más frecuentes fueron diabetes, hipertensión y obesidad). Más de la mitad de los casos fueron traslados de otros hospitales, el $73 \%$ habían
Tabla 4. Características del derrame pleural paraneumónico o del empiema en la población estudiada

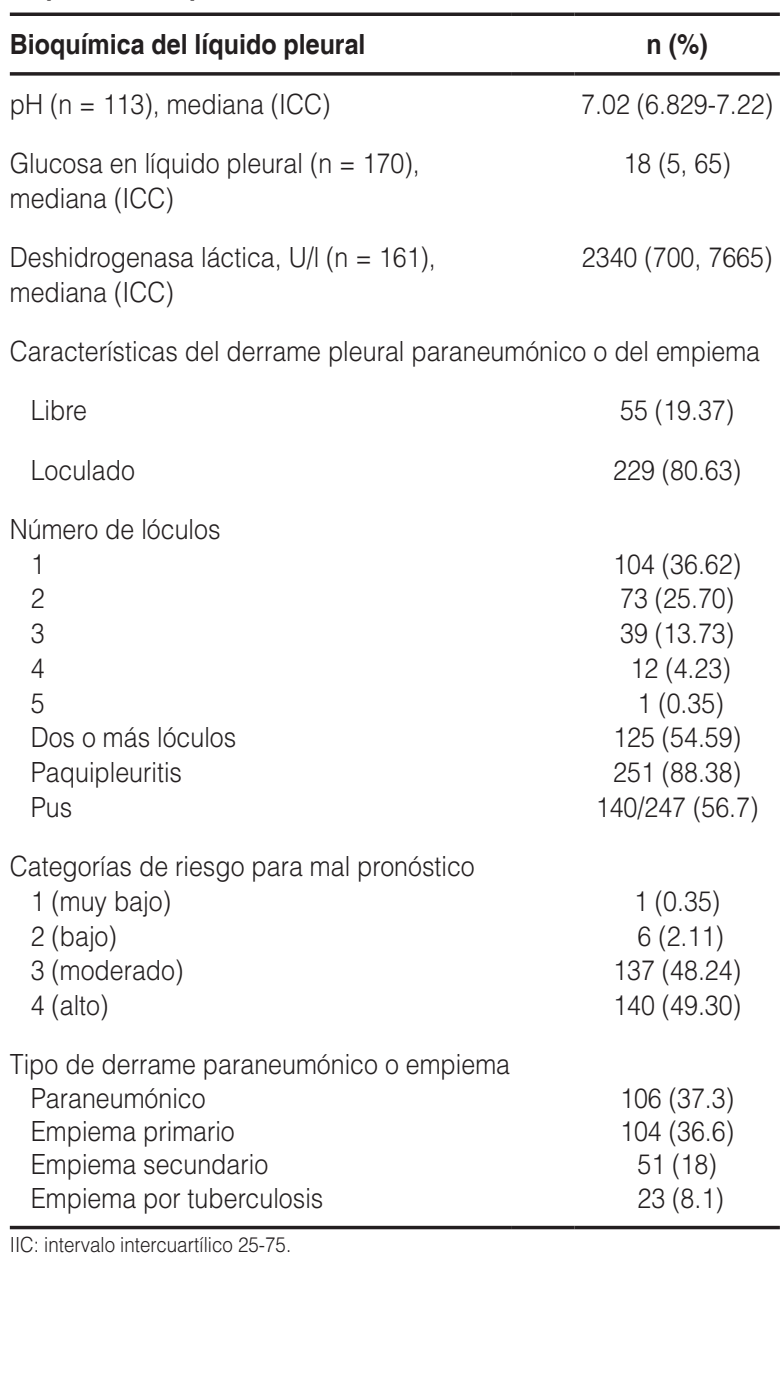

recibido antibiótico previo al ingreso al hospital y el $54 \%$ no se resolvió con drenaje torácico mediannte sonda endopleural y antibióticos, por lo que requitirieron cirugía, que en el $90 \%$ de los pacientes consistió en lavado de la cavidad y decorticación.

Otros estudios informan de un predominio de hômbres semejante al de nuestro estudio y una mediana de edad de 57 años, que es mayor que la de nuestra población (47 años)1.

En un estudio sobre los factores de riesgo pará el desarrollo de DPP/E, la frecuencia y el tipo de comorbilidad fue muy variable'. La diabetes fue mucho nos frecuente que en nuestro estudio ( 6.5 vs. $35 . \overline{6} \%$ ) y no se reportó hipertensión arterial sistémica ni obesidad, que fueron otras de las afecciones más frecuentes en nuestro estudio, pero sí se describe enfermedad hepática o cardiaca con una frecueñcia de aproximadamente el $10 \%$. 
Tabla 5. Microorganismos más frecuentemente identificados en el líquido pleural de 89 pacientes con derrame paraneumónico y empiema

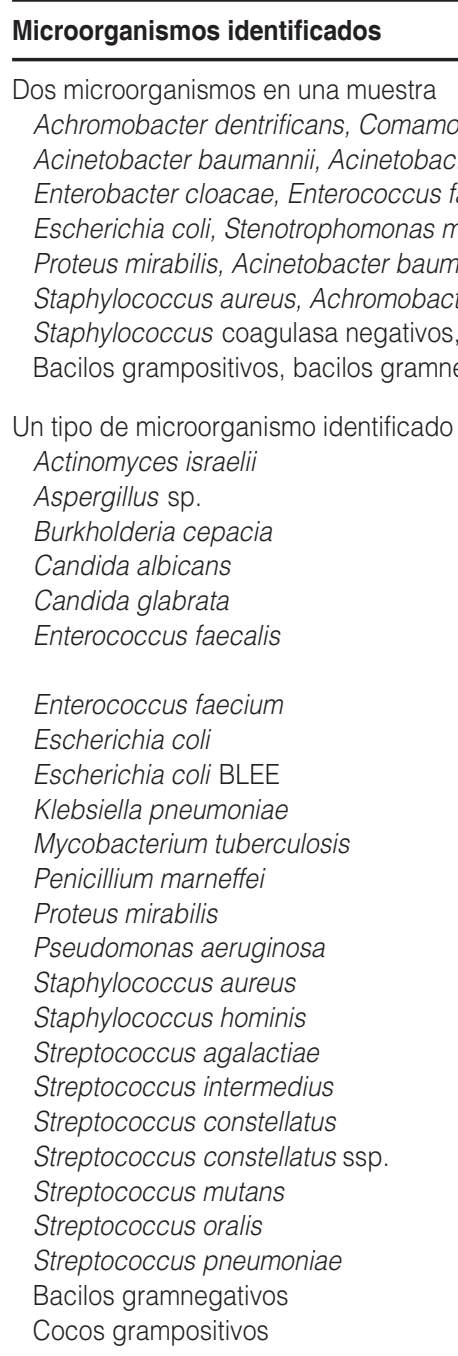

Principales especies de bacterias gramnegativas identificadas: Escherichia coli, Enterococcus faecalis, Enterococcus faecium, Pseudomonas aeruginosa, Acinetobacter baumannii, Klebsiella pneumoniae, etc.

Principales especies de bacterias grampositivas identificadas: Staphylococcus aureus, Streptococcus constellatus,

Streptococcus pneumoniae, etc.

Cocos grampositivos y bacilos grampositivos y gramnegativos sin identificación de especie

Hongos: Candida albicans, Aspergillus sp., Penicillium marneffel)

Mycobacterium tuberculosis

Los síntomas más frecuentes en la población estudiada fueron tos con expectoración, fiebre, dolor torácico tipo pleurítico y disnea, mismos que son

1

1

1

1

1

1

Tabla 6. Frecuencia y tipo de cirugía realizada en los pacientes con derrame pleural paraneumónico o empiema

n

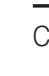
con derrame pleural paraneumónico o empiema 
intervalo que se describe en la literatura. La explicación a este alto número de cultivos negativos tiene varias razones; entre ellas, que no se cultivó para microorganismos anaerobios ni se utilizaron métodos moleculares adicionales a los medios tradicionales de cultivo para el presente estudio, y por otra parte, una proporción muy elevada de pacientes $(72.9 \%)$ habían recibido tratamiento antibiótico previo a la toma de la muestra de líquido pleural. Adicionalmente, es posible que en algunos casos se haya obtenido líquido inflamatorio estéril adyacente a un lóculo con bacterias.

Hay varias técnicas quirúrgicas que se han empleado para el tratamiento del DPP/E, incluyendo la decorticación pleural, la toracoscopia videoasistida (TVA), la toracoplastia y la pleurotomía abierta tipo Eloesser ${ }^{8}$.

En el 54\% de nuestros casos se practicó cirugía, cuyo tipo más frecuente fue la toracotomía con decorticación, en el $90 \%$ de las ocasiones. Debido al momento tardío en que llegaron la mayoría de nuestros pacientes, es evidente que presentaron las complicaciones esperadas por la etapa de organización del empiema, como son la formación de lóculos y paquipleuritis $^{19}$ que condicionaron, por un lado, el drenaje incompleto de la cavidad, y por otro, el atrapamiento pulmonar, situaciones que muchas veces solo se pueden resolver mediante toracotomía y decorticación ${ }^{20}$, procedimiento que resuelve la sepsis pleural y permite que el pulmón se expanda al liberarlo de las adherencias pleurales, mejorando ostensiblemente la capacidad vital forzada y el volumen espiratorio forzado al primer segundo. A pesar de que se trata de una cirugía mayor, la mediana de la estancia hospitalaria posoperatoria no es tan prolongada; por ejemplo, en el estudio de Pothula y Krellenstein ${ }^{7,21}$ fue de 7 días, aunque mucho menor comparada con la estancia hospitalaria de nuestros pacientes, que fue de 11 (8-20) días.

El desbridamiento por TVA ha ganado popularidad desde mediados de la década de $1990^{3}$, por lo que desde hace tiempo esta técnica se ha propuesto para los casos complicados con drenaje incompleto de la cavidad pleural, y ha demostrado ser de utilidad para el desbridamiento en el empiema multiloculado o con un lóculo, que no se resuelve con tratamiento antibiótico y drenaje $e^{22}$. Sin embargo, en nuestros casos fue el procedimiento que menos se realizó (1.96\%), debido a la etapa de presentación tan avanzada de los pacientes, pues el $97.5 \%$ presentaron categorías de riesgo elevado para complicaciones ${ }^{3}$. La evidencia previa obtenida en estudios retrospectivos sobre la eficacia de la TVA como método definitivo de tratamiento en estos casos es variable, desde un $56 \%$ hasta un $97 \%^{11,23}$, y es altamente dependiente de la etapa en que se encuentra el DPP/E: cuanto más avanzada la fase de organización, mayor frecuenccia de falla. Por otra parte, cuando se comparó esta đécnica con la terapia fibrinolítica en un ensayo clínico aleatorizado en pacientes con DPP/E loculado se demostró que la TVA fue más eficaz que la terapia fibrinolítica y se asoció con un menor tiempo deestancia hospitalaria y un menor costo ${ }^{24}$.

La TVA es un procedimiento quirúrgico de invasión mínima que puede resolver el empiema en los casos que aún no son crónicos; sin embargo, se sugiere gue se puede escoger libremente el realizar una toracotomía en los casos crónicos, en especial en aquêllos pacientes que tardan en ser referidos más de 2 semmanas y cuando se documentan microorganismos grámnegativos como causantes de la enfermedad ${ }^{18}$, como sucedió en nuestros casos, cuya mediana del tiempo del padecimiento actual fue de 24 días y predominăron las bacterias gramnegativas. No obstante, es pertinente comentar que este último criterio no se tomó en cuenta para decidir el tipo de abordaje quirúrgico

El tratamiento definitivo de los pacientes con DPP/E debe realizarse dentro de los primeros 10 días de hospitalización ${ }^{11}$, lo que en nuestra serie ocurrió el el $59 \%$ de los casos, aunque la mediana (ICC) de tièmpo transcurrido entre el ingreso del paciente y etódía en que se practicó la cirugía fue de 8 (4-14) días.Sin embargo, la mortalidad hospitalaria fue del $5.63 \%$, cifra que se ubica en el límite inferior de la mortalidad reportada en otros estudios de décadas pasadas:-que es del $4-24 \% \%^{18,25}$; así mismo, esta ocurrió en la mayoría de los casos de etiología bacteriana, pues solo entuna de las 16 defunciones el paciente tenía tuberculoșis.

En ninguno de nuestros pacientes se utilizó teräpia fibrinolítica, hecho que consideramos que no inftuyó sobre la elevada frecuencia de cirugía para resolver la infección pleural. Se ha descrito que la utilización de fibrinolíticos intrapleurales no es un tratamiento sistemático para el DPP/E debido a que no ha demostrado beneficio sobre la reducción de la mortalidäd ${ }^{26}$, y los resultados de un ensayo clínico realizado ên el año 2005, que incluyó 427 pacientes, tampoco çdemostraron beneficio sobre la mortalidad, la tasa de cirugía ni el tiempo de estancia hospitalaria ${ }^{27}$.

\section{Conclusiones}

La mayor parte de los pacientes llegaron en etâpas avanzadas de la enfermedad, y como ello va acorde 
con el tiempo de evolución del padecimiento, más de la mitad de los casos requirieron cirugía para resolver la enfermedad, de los cuales en el $90 \%$ fue toracotomía con lavado y decorticación. Los resultados del presente estudio reflejan la necesidad de favorecer mecanismos para realizar un diagnóstico y un tratamiento tempranos en los pacientes con DPP/E, con la finalidad de disminuir la necesidad de tratamiento quirúrgico $\mathrm{y}$, si este es necesario, aumentar la frecuencia con que se practica TVA.

\section{Conflicto de intereses}

Los autores declaran que no existe conflicto de intereses.

\section{Responsabilidades éticas}

Protección de personas y animales. Los autores declaran que para esta investigación no se han realizado experimentos en seres humanos ni en animales.

Confidencialidad de los datos. Los autores declaran que en este artículo no aparecen datos de pacientes.

Derecho a la privacidad y consentimiento informado. Los autores declaran que en este artículo no aparecen datos de pacientes.

\section{Bibliografía}

1. Chalmers JD, Singanayagam A, Murray MP, Scally C, Fawzi A, Hill AT. Risk factors for complicated parapneumonic effusion and empyema on presentation to hospital with community-acquired pneumonia. Thorax. 2009;64:592-7.

2. Falguera M, Carratalá J, Bielsa S, García-Vidal C, Ruiz-González A Chica I, et al. Predictive factors, microbiology and outcome of patients with parapneumonic effusion. Eur Respir J. 2011:38:1173-9.

3. Feller-Kopman D, Light R. Pleural disease. N Engl J Med. 2018;378:740-51.

4. Lehtomäki A, Nevalainen R, Ukkonen M, Nieminen J, Laurikka J, Khan J. Trends in the incidence, etiology, treatment, and outcomes of pleural infections in adults over a decade in a Finnish university hospital. Scand J Surg. 2020;109:127-32.

5. Søgaard M, Nielsen RB, Nørgaard M, Kornum JB, Schønheyder HC, Thomsen RW. Incidence, length of stay, and prognosis of hospitalized patients with pleural empyema: a 15-year Danish nationwide cohort study. Chest. 2014;145:189-92.
6. Nayak R, Lougheed MD, Brogly S, Lajkosz K, Petsikas D. Thoracic empyema -20 year trends in management and outcomes in Ontario, Canada. Am J Respir Crit Care Med. 2018;197:A4215.

7. Grijalva CG, Zhu Y, Nuorti JP, Griffin MR. Emergence of parapneumonic empyema in the USA. Thorax. 2011;66:663-8.

8. Semenkovich TR, Olsen MA, Puri V, Meyers BF, Kozower BD. Current state of empyema management. Ann Thorac Surg. 2018;105:1589-96.

9. Reichert M, Hecker M, Witte B, Bodner J, Pdberg W, Weigand MAEet al. Stage-directed therapy of pleural empyema. Langenbecks Arch Surg. 2017;402:15-26

10. Herrera-Kiengelher L, Báez-Saldaña R, Salas-Hernández J, AvaloßBracho A, Pérez-Padilla R, Torre-Bouscoulet L. Frequency of adverse events and mortality in patients with pleural empyema in a public referral hospital in Mexico City. Int J Tuberc Lung Dis. 2010;14:1187-92.

11. Molnar TF. Current surgical treatment of thoracic empyema in adults Eur J Cardiothorac Surg. 2007;32:422-30.

12. World Health Organization. Physical status: The use and interpretation of anthropometry. World Health Organization Technical report series 854 Geneva: WHO; 1995. (Consultado el 24 de octubre de 2018.) Disponible en: https://apps.who.int/iris/bitstream/handle/10665/37003/WHO_ TRS_854.pdf.

13. Ahmed RA, Marrie TJ, Huang JQ. Thoracic empyema in patients with community-acquired pneumonia. Am J Med. 2006;119:877-83.

14. Burgos J, Luján M, Falcó V, Sánchez A, Puig M, Borrego A, et al. The spectrum of pneumococcal empyema in adults in the aearly 21 st century. Clin Infect Dis. 2011;53:254-61.

15. Clark J. Microbiology and management of pleural empyema. AdV Exp Med Biol. 2009;634:61-8.

16. Maskell NA, Batt S, Hedley EL, Davies CW, Gillespie SH, Davies RJ.The bacteriology of pleural infection by genetic and standard methods and its mortality significance. Am J Respir Crit Care Med. 2006;174:817-23.

17. Marks DJB, Fisk MD, Koo CY, Pavlou M, Peck L, Lee SF, et al. Thoracic empyema: a 12-year study from a UK tertiary cardiothoracic referral centre. PLoS One. 2012;7:e30074.

18. Lardinois D, Gock M, Pezzetta E, Buchli C, Rousson V, Furrer M,cet al. Delayed referral and gram-negative organisms increase the conversion thoracotomy rate in patients undergoing video-assisted thoracoscopic surgery for empyema. Ann Thorac Surg. 2005;79:1851-6.

19. Mutsaers SE, Prele CM, Brody AR, Idell S. Pathogenesis of pleural fibrosis. Respirology. 2004:9:428-40.

20. Rzyman W, Skokowski, Romanowicz G, Lass P, Dziadziuszko R. Decortication in chronic pleural empyema - effect on lung function. Eur $J$ Cardiothorac Surg. 2001;21:502-7.

21. Pothula V, Krellenstein DJ. Early aggressive surgical management of paraneumonic empyemas. Chest. 1994;105:832-6.

22. Corcoran JP, Wrightson JM, Belcher E, DeCamp MM, Feller-Kopman D, Rahman NM. Pleural infection: past, present, and future directions. Lancet Respir Med. 2015;3:563-77.

23. Reichert M, Pösentrup B, Hecker A, Schneck E, Pons-Kühnemann J, Augustin $\mathrm{F}$, et al. Thoracotomy versus video-assisted thoracoscopic surgery (VATS) in stage III empyema-an analysis of 217 consecutive patients. Surg Endosc. 2018;32:2664-75.

24. Wait MA, Sharma S, Hohn J, Dal Nogare A. A randomized trial ofempyema therapy. Chest. 1997;111:1548-51.

25. Park C-K, Oh H-J, Choi H-Y, Shin HJ, Lim JH, Oh IJ, et al. Microbiological characteristics and predictive factors for mortality in pleural infection: a single-center cohort study in Korea. PLoS One 2016:11:e0161280.

26. Altmann ES, Crossingham I, Wilson S, Davies HR. Intra-pleural fibrinolytic therapy versus placebo, or a different fibrinolytic agent, in the treatment of adult parapneumonic effusions and empyema. Cochrane Database Syst Rev. 2019;(10):CD002312.

27. Cameron R, Davies HR. Intra-pleural fibrinolytic therapy versus conservative management in the treatment of adult parapneumonic effusions and empyema. Cochrane Database Syst Rev. 2008;(16):CD002312.

28. Maskell NA, Davies CW, Nunn AJ, Hedley EL, Gleeson FV, MillerR, et al. U.K. Controlled trial of intrapleural streptokinasa for pleural infection. N Engl J Med. 2005;352:865-874. DOI: 10.1056/NEJMoa042473든 